\title{
Crystal structures, magnetic and superconducting properties of the $\mathrm{RuSr}_{2} \mathrm{NdCu}_{2} \mathrm{O}_{x}$ and $\mathrm{RuSr}_{2} \mathrm{GdCu}_{2} \mathrm{O}_{y}$ compounds
}

November 19, 2018

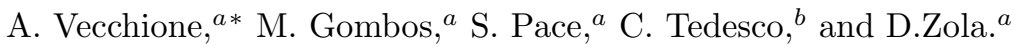

${ }^{a}$ Unità I.N.F.M. di Salerno, Dipartimento di Fisica "E.R. Caianiello"

Università di Salerno, Via S. Allende, I-84081 Baronissi (Salerno), Italy

${ }^{b}$ Dipartimento di Chimica, Università di Salerno, via S. Allende, I-84081 Baronissi, Italy

\begin{abstract}
We report the magnetization and the susceptibility measurements of the $\mathrm{RuSr}_{2} \mathrm{GdCu}_{2} \mathrm{O}_{y}$ and $\mathrm{RuSr}_{2} \mathrm{NdCu}_{2} \mathrm{O}_{x}$ perovskite materials. We find that $\mathrm{RuSr}_{2} \mathrm{GdCu}_{2} \mathrm{O}_{y}$ compound exhibits a magnetic transition at $T_{n}=135$ $\mathrm{K}$ followed by a superconducting one with an onset $T_{c}=35 \mathrm{~K}$. Samples of $\mathrm{RuSr}_{2} \mathrm{NdCu}_{2} \mathrm{O}_{x}$ material have shown neither superconductivity nor magnetic transition. XRD measurements show that in $\mathrm{RuSr}_{2} \mathrm{NdCu}_{2} \mathrm{O}_{x}$ the $\mathrm{Nd}$ ions tend to substitute to $\mathrm{Sr}$ ions in very high percentages. The relevance of this phenomenon on the absence of superconductivity in $\mathrm{RuSr}_{2} \mathrm{NdCu}_{2} \mathrm{O}_{x}$ is discussed.
\end{abstract}

Keywords: Rutheno-cuprate materials, Superconductivity, Magnetization, $\mathrm{X}$-ray powder diffraction.

*Antonio Vecchione, Unità I.N.F.M. di Salerno, Dipartimento di Fisica "E.R. Caianiello", Università di Salerno, Via Salvador Allende, I-84081 Baronissi (Salerno), Italy. Fax: +39089 953804, e-mail: vecchione@sa.infn.it 
The rutheno-cuprate $\mathrm{RuSr}_{2} \mathrm{GdCu}_{2} \mathrm{O}_{y}(\mathrm{Gd}-1212)$ system is a subject of great interest because exhibits coexistence of bulk superconductivity and magnetic ordering [1]. The $\mathrm{RuSr}_{2} \mathrm{GdCu}_{2} \mathrm{O}_{y}$ tetragonal structure is an analog of $\mathrm{Y}_{1} \mathrm{Ba}_{2} \mathrm{Cu}_{3} \mathrm{O}_{7}$ with replacement of $\mathrm{Ru}$ ions into $\mathrm{Cu}(1)$ sites. This results in a perovskite consisting of different sequences of alternating $\mathrm{CuO}_{2}$ bilayers and $\mathrm{RuO}_{2}$ monolayer. In this hybrid rutheno-cuprate system both the $\mathrm{Cu}-\mathrm{O}$ and $\mathrm{Ru}-\mathrm{O}$ planes form very similar square-planar arrays, and the coexistence of superconductivity and long range magnetic order is intriguing [2]. However, in spite of extensive investigation a consistent picture of the magnetic structure is still lacking [3], 伍.

In the present investigation, we show the effects on the structural and superconducting properties of Gd-1212 rutheno-cuprate compound by substituting Gd rare earth with $\mathrm{Nd}$.

Policrystalline samples of $\mathrm{RuSr}_{2} \mathrm{NdCu}_{2} \mathrm{O}_{x}$ and $\mathrm{RuSr}_{2} \mathrm{GdCu}_{2} \mathrm{O}_{y}$ were prepared by conventional solid state reaction by mixing stoichiometric amounts of highly pure $\mathrm{RuO}_{2}, \mathrm{Nd}_{2} \mathrm{O}_{3}, \mathrm{Gd}_{2} \mathrm{O}_{3}, \mathrm{CuO}$ oxides and strontium carbonate $\left(\mathrm{SrCO}_{3}\right)$. After calcination in air at $960^{\circ} \mathrm{C}$ the powders were ground, milled and annealed in argon flow at $1020^{\circ} \mathrm{C}$ for 10 hours. At the final stage the powders were oxygenated with a procedure made by several cycles of heating at temperatures ranging from $1050^{\circ} \mathrm{C}$ to $1080{ }^{\circ} \mathrm{C}$. The phases produced after each step were monitored by X-ray diffraction.

X-ray diffraction patterns were recorded by means of Philips PW-1700 diffrac- 
tometer using Ni-filtered $\mathrm{Cu} \mathrm{K} \alpha$ radiation. Magnetization and susceptibility measurements were performed by means of a Oxford Maglab Vibrating Sample Magnetometer (VSM). The samples vibrate in a region where the field homogeneity is equal to $10^{-6}$. Structural and magnetization measurements were carried out after sieving the materials and collecting the fraction with size smaller than $10 \mu \mathrm{m}$. The correct stoichiometry of both $\mathrm{RuSr}_{2} \mathrm{NdCu}_{2} \mathrm{O}_{x}$ and $\mathrm{RuSr}_{2} \mathrm{GdCu}_{2} \mathrm{O}_{y}$ compounds and the absence of contamination from both alumina crucibles and other spurious elements were checked by EDS analysis.

X-ray diffraction patterns of oxygen annealed $\mathrm{RuSr}_{2} \mathrm{NdCu}_{2} \mathrm{O}_{x}$ and $\mathrm{RuSr}_{2} \mathrm{GdCu}_{2} \mathrm{O}_{y}$ samples are shown in Fig.1. Reflections marked by stars are due to small amounts of extra-phase. X-ray diffraction patterns of $\mathrm{RuSr}_{2} \mathrm{NdCu}_{2} \mathrm{O}_{x}$ has been successfully indexed assuming a cubic cell with $a=3.907 \AA$. This implies that a disordered cubic perovskite structure can be assumed: $\mathrm{Nd}$ and $\mathrm{Sr}$ cations occupy the same site and the same applies to $\mathrm{Cu}$ and $\mathrm{Ru}$. As already reported [2], $\mathrm{RuSr}_{2} \mathrm{GdCu}_{2} \mathrm{O}_{y}$ exhibits a tetragonal cell $(a=3.838 \AA, c=11.573 \AA)$ with alternating $\mathrm{CuO}_{2}$ and $\mathrm{RuO}_{2}$ planes. The proposed structural model for Nd1212 compound in space group $P m \overline{3} m$ is similar to that of $\mathrm{RuSr}_{2} \mathrm{GdCu}_{2} \mathrm{O}_{y}$ with mixed $\mathrm{Cu} / \mathrm{Ru}$ and $\mathrm{Nd} / \mathrm{Sr}$ cation sites and oxygen coordinates fixed by the symmetry of the space group.

The possible effects of the cations intermixing on the magnetic and superconducting properties were analyzed by susceptibility and magnetization measurements. We measured a DC susceptibility, $\chi$, for $\mathrm{RuSr}_{2} \mathrm{NdCu}_{2} \mathrm{O}_{x}$ and 
$\mathrm{RuSr}_{2} \mathrm{GdCu}_{2} \mathrm{O}_{y}$ samples in the temperature range from $5 \mathrm{~K}$ to $270 \mathrm{~K}$. In Fig.2 we show the zero-field-cooled DC susceptibility, $\chi(T)$, measured in $\mathrm{RuSr}_{2} \mathrm{NdCu}_{2} \mathrm{O}_{x}$ powders in an external field of $10 \mathrm{G}$. In the temperature range investigated neither ferromagnetic nor superconducting transition occurs. The $\mathrm{RuSr}_{2} \mathrm{NdCu}_{2} \mathrm{O}_{x}$ is paramagnetic and follows a behaviour well described in term of Curie law for $T<150 \mathrm{~K}$ (see inset of Fig.2). We also carried out measurements of $\chi(T)$, shown in the inset of the Fig.3, on a $\mathrm{RuSr}_{2} \mathrm{GdCu}_{2} \mathrm{O}_{y}$ sample. The main features observed are a large rise for $T<150 \mathrm{~K}$ due to the magnetic transition and a large drop for $T<35 \mathrm{~K}$ ascribed to the onset superconducting transition. The signal revealed in superconducting region is not diamagnetic and no Meissner phase is observed down to $5 \mathrm{~K}$ as already reported in literature [1, 5]. We do not observe a peak in DC susceptibility. A reason could be in our experimental set-up. In fact the superconducting magnet of the VSM has a persistent field measured by Hall probe which has $1 \mathrm{G}$ as sensibility. To perform zero field cooling measurement we cancel this persistent field applying an opposite magnetic field. Probably the residual field lower than $1 \mathrm{G}$ is enough to orientate the magnetic domain in our sample during the initial cooling. However, the peak in zero field cooled susceptibility was not observed by Felner et al. in not superconducting $\mathrm{RuSr}_{2} \mathrm{EuCu}_{2} \mathrm{O}_{8}$ and by Williams and Kramer for field higher than $2.5 \mathrm{kG}$ [6, 可].

In order to investigate the magnetic behaviour in the $\mathrm{RuSr}_{2} \mathrm{GdCu}_{2} \mathrm{O}_{y}$ sample, we performed measurements of the magnetization $M$ in external magnetic field 
$H$ up to $5 \mathrm{~T}$ at different temperatures. As can be observed from Fig. 3, an irreversible behaviour is revealed in $M(H)$ loop. This finding has been ascribed [1] to the presence of a ferromagnetic phase in these samples.

Our data point out that the substitution of the neodymium in Gd-1212 compounds involves a change in the superconducting and magnetic properties. The observed magnetic transition in $\mathrm{RuSr}_{2} \mathrm{GdCu}_{2} \mathrm{O}_{y}$, attributed to the ordering in the ruthenium planes, does not take place in $\mathrm{RuSr}_{2} \mathrm{NdCu}_{2} \mathrm{O}_{x}$. At the same time the superconducting transition, occurring in copper planes of Gd-based compound, is also suppressed. The presence of structural disorder in Nd-based compound play a role preventing a long range extension of both $\mathrm{Cu}-\mathrm{O}$ and $\mathrm{Ru}-\mathrm{O}$ planes through out the structure. This implies the suppression of the superconducting and (ferro)magnetic order parameters.

In conclusion, we have prepared $\mathrm{RuSr}_{2} \mathrm{NdCu}_{2} \mathrm{O}_{x}$ and $\mathrm{RuSr}_{2} \mathrm{GdCu}_{2} \mathrm{O}_{y}$ materials whose structure and transport properties have been described by XRD analysis and magnetization and susceptibility measurements. A description of the x-ray patterns has been achieved assuming that the Nd-based 1212 phase is cubic and not isostructural to the Gd-1212 phase. In particular, we attribute the absence of superconductivity and magnetic ordering in $\mathrm{RuSr}_{2} \mathrm{NdCu}_{2} \mathrm{O}_{x}$ compound to the partial intermixing between $\mathrm{Ru}$ and $\mathrm{Cu}$ and between $\mathrm{Nd}$ and $\mathrm{Sr}$ into the crystalline structure.

We acknowledge Dr. Canio Noce for helpful discussions.

\section{References}


[1] C. Bernhard et al., Phys. Rev. B 59, 14099 (1999).

[2] A. C. McLaughlin et al., Phys. Rev. B 60, 7512 (1999).

[3] A. Fainstein et al., Phys. Rev. B 60, 12597 (1999); A. C. McLaughlin and J. P. Attfield, Phys. Rev. B 60, 14605 (1999).

[4] J.W. Lynn et al., Phys Rev B 61, 14964 (2000).

[5] C.W. Chu et al. Physica C 335, 231 (2000)

[6] I. Felner, U. Asaf, Y. Levi, and O. Millo, Phys. Rev. B 55, 3374 (1997); E. B. Sonin and I. Felner, Phys. Rev. B 57, 14000 (1998); I. Felner, U. Asaf, S. Reich, and Y. Tsabba, Physica C 311, 163 (1999); I. Felner, U. Asaf, Y. Levi, and O. Millo, Physica C 334, 141 (2000).

[7] G. V. M. Williams and S. Krämer, Phys. Rev. B 62, 4132 (2000). 


\section{Figure Captions}

Figure 1. X-ray diffraction powder patterns of the $\mathrm{RuSr}_{2} \mathrm{NdCu}_{2} \mathrm{O}_{x}$ (up) and $\mathrm{RuSr}_{2} \mathrm{GdCu}_{2} \mathrm{O}_{y}$ (bottom). Peaks marked by stars are due to phase impurities.

Figure 2. DC susceptibility measurement of the $\mathrm{RuSr}_{2} \mathrm{NdCu}_{2} \mathrm{O}_{x}$ compound. Inset: $1 / \chi$ versus $T$ showing Curie paramagnetic behaviour.

Figure 3. Magnetization measurements of $\mathrm{RuSr}_{2} \mathrm{GdCu}_{2} \mathrm{O}_{y}$ taken at three different temperatures. Inset: DC susceptibility tversus $T$ measurement. 







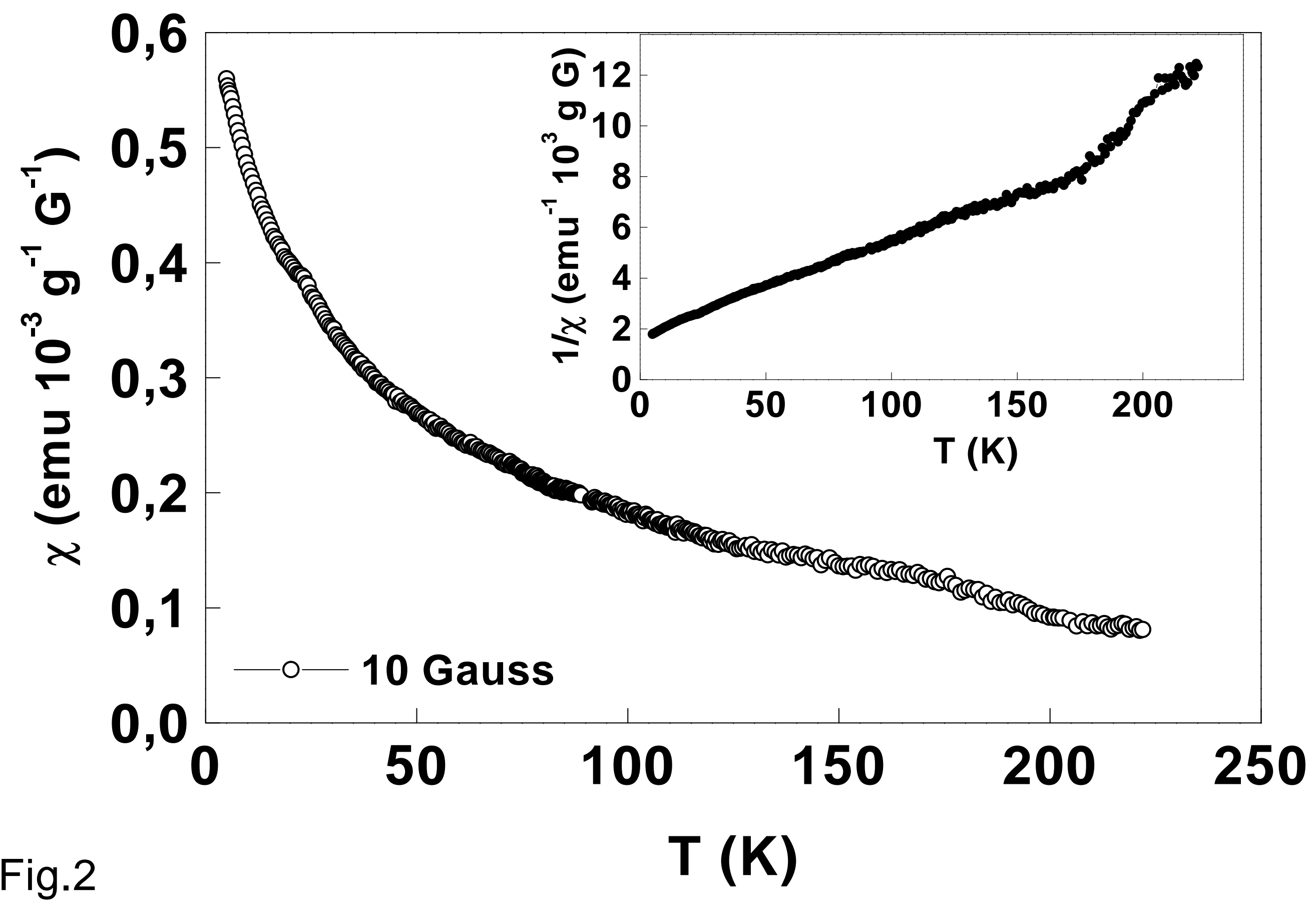




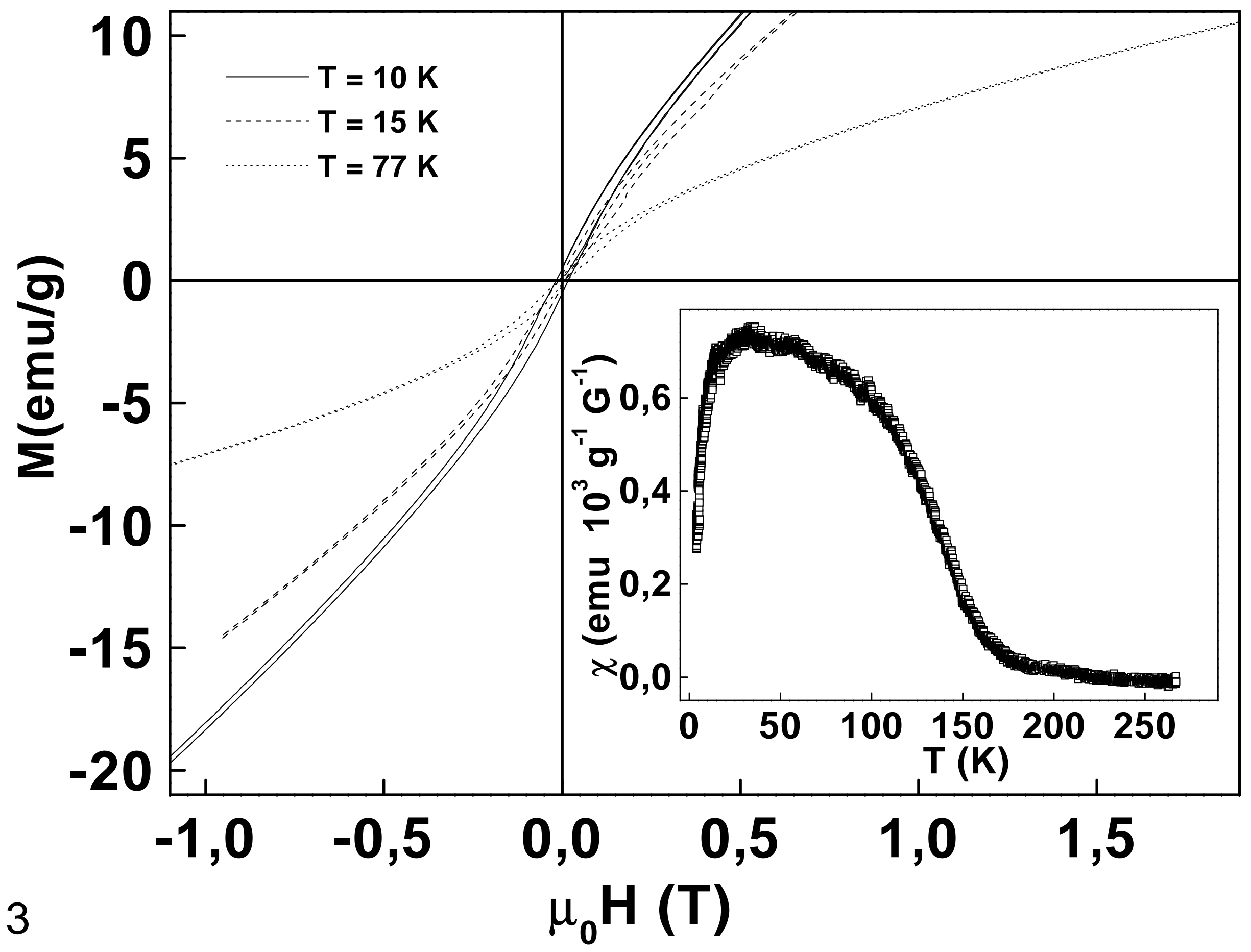

Fig. 3 